\title{
Modular testbed for spinning spacecraft
}

\begin{abstract}
The ground verification of a spacecraft control algorithm is commonly done via air bearing facility. Air bearing testbeds are frequently developed for testing a three-axis stabilised spacecraft control algorithm but hardly for a spin stabilised spacecraft. A modular testbed for testing a spinning spacecraft has been developed in the Surrey Space Centre (SSC) initially for the real-time verification of a prolate spinner slew control algorithms. This testbed is made from commercially-offthe-shelf (COTS) components with a modular system design approach through rapid control prototyping (RCP) using Matlab xPC Target and extendable to other RCP technique. It is equipped with a novel low cost monocular vision system for attitude determination with the accuracy of $0.06 \mathrm{deg}$ and angular velocity accuracy of $0.15 \mathrm{deg} / \mathrm{s}$. For the current specification, a cold gas propulsion system is fixed to the testbed with a 2-DOF thruster set that can deliver up to $0.25 \mathrm{~N}$ of thrust and an air bearing capability that gives 3-DOF with a maximum tilt angle of $30 \mathrm{deg}$. In this paper, the testbed implementation is described and the test platform is verified.
\end{abstract}

Index Terms - testbed, spin-stabilised spacecraft, modular, ground testing, rapid control prototyping

\section{INTRODUCTION}

$\mathrm{G}$ ROUND verification is a crucial step towards producing a working spacecraft especially for its attitude control and determination subsystem (ADCS). The usage of an air bearing platform is a common practice nowadays in realizing this goal. However as the trend for spacecraft stabilization leans towards three-axis stabilization, majority of the ground verification air bearing testbeds have been developed to serve this purpose. Surrey Space Centre (SSC) has recently developed a testbed for a spin-stabilized spacecraft using a spherical air bearing platform. This testbed was initially targeted to be used as a real-time verification tool for the state-of-the-art slew control algorithms of prolate spinner [1-3]. This prolate spinner or spin-stabilized spacecraft was intended for the moon penetrator mission called MoonLITE [4].

The design of the testbed has been made modular both physically and systematically. A square aluminum frames with a width and length of $15 \mathrm{~cm}$ and height that varies are used to house the required subsystems on top of the air bearing platform. These frames are arranged according to the type of verification that needed to be done using the testbed. For example, they are arranged vertically to produce a shape that is prolate for the purposes of testing a slew control algorithms for prolate spinner. Small form factor computer is used as the on-board computer (OBC) of the testbed. Combining this with a Matlab ${ }^{\circledR}$ XPC Target rapid control prototyping (RCP) approach, a modular system design is achieved for the testbed. Any intended control algorithm that normally developed using a Simulink environment can be uploaded to this testbed OBC for real time verification.

The testbed is equipped with a novel low-cost monocular vision system [5] for its attitude determination. The vision system utilizes five infra-red LED (IrLED) markers mounted on the testbed platform with a typical USB camera installed above it using an isolated frame. This camera that is equipped with an infra-red filter will acquire the real-time position of these markers. The position data will be compared to the markers' initial position stored in the system and the current attitude of the testbed is computed. The attitude accuracy achievable by the vision system is $0.06 \mathrm{deg}$ while the velocity accuracy is measured at $0.15 \mathrm{deg} / \mathrm{s}$ [5]. This velocity accuracy however can be improved by utilizing a camera that has a faster refresh rate than the existing unit.

For attitude actuation, the testbed is equipped with a coldgas propulsion system. This propulsion system will enable the testbed to spin in one axis in order to create a spin-stabilized testing platform before any needed control algorithm can be tested. Commercially-off-the-shelf (COTS) solenoid valves are used as the thruster and with the current configuration, the thruster can produce up to $0.25 \mathrm{~N}$ of thrust. The propulsion system can be easily modified to suit certain application. For the current arrangement additional thruster is placed in an orthogonal axis to the spin axis to demonstrate the slew control algorithms for spinner that are currently being tested.

Other details of the testbed design are further described in this paper that is organised in five sections. The next Section II provides survey of the current testbeds trend. This section also lists the design concept that is used as a guideline to produce the final testbed design. This final testbed design is then described in the following Section III, where the design trade-off and the hardware setup are discussed. Section IV presents the preliminary analysis of the testbed performance via a series of spin test and payload integration that leads to the testbed profile and the demonstration of its system modularity. Finally Section V describes some conclusions of the development work and recommendation to improve this unique testbed for future use.

\section{CurRent TREnd \& Design CONCEPT}

The usage of testbeds to verify the attitude control mechanism of spacecraft is not a new trend as this has been done as early as the beginning of spaceflight itself. The 
implementation of an air bearing platform for this specific purpose has started for half a century ago [6]. The reason for such a testbed is to make sure that the attitude control of a spacecraft is thoroughly verified before the mission in space begins. The failure in the attitude control will result in a mission failure in most of the space mission. The following sub-sections will briefly review the related testbed and the design concept extracted from the review before the final testbed setup is implemented.

\section{A. Current Testbed Survey}

A high-end multipurpose spacecraft attitude control testbed is reviewed firstly to get a latest trend in ground testing for satellite attitude control. As the name indicates, this high-end platform is an expensive solution hence defying the first intention of the design to have a low cost implementation by utilising COTS components. Setting aside this difference, the purpose of reviewing this testbed is to highlight a feature that can be used in developing the intended testbed. One example is the ACS Test Stand developed in Astro- und Feinwerktechnik Adlershof GmbH, Berlin, Germany.

The details for the mentioned testbed can be acquired from Ref. 7. The inclusion of the magnetic field emulator and the sun simulator in this testbed can be the possible added features to this developed platform. This will help in testing algorithms that are dependent to such factors such as the Rhumb Line slew algorithm for the prolate spinner or the algorithms that use a magnetorquer as the actuator.

A testbed for spacecraft control is not only confined within a ground testing facility. A low cost cubesat platform can also be used. For example, the Surrey Training, Research and Nanosatellite Development (STRaND) was proposed to be used as the testing platform for the developed pulse plasma thruster (PPT) and verification of the spinner slew control algorithm [8]. Through this programme, a 3-U cubesat that housed novel low cost space technologies has been launched in Febraury 2013 with the mission name STRaND-1.

As the satellite has already been launched, modification to the system cannot be made to accommodate any further needs. The ground space testing facility would easily accommodate this problem hence it is preferable compare to the satellite based testing platform such as the reviewed STRaND-1.

Apart from the mentioned testbed, there are also facilities designed specifically for a unique purpose. Ref. $9-11$ are some examples of those testbeds where the design are made towards certain applications. The different approaches in the implementation gave each and every testbed its own identity and novelty in handling the challenges identified during the development. This is the case for the spinning testbed as the stabilizing technique is different from the typical 3-axis stabilized method.

Following the survey on the available testbed above, a survey on a suitable testbed platform is also made to strengthen the literature. Table 1 below summarises all usable platform that can be utilised in developing the testbed. It clearly shows that the air bearing platform is the best medium for carrying out the needed verification test.

TABLE 1

SURVEY ON TESTING MEDIUM

\begin{tabular}{|c|c|c|c|c|c|}
\hline \multirow[b]{2}{*}{ Limiting Factor } & \multicolumn{5}{|c|}{ Testbed Facility } \\
\hline & $\begin{array}{l}\text { Spherical Air-Bearing } \\
\text { Platform }\end{array}$ & Parabolic Flight ${ }^{\text {b }}$ & Drop Tower " & On-orbit spacecraft & Space Station \\
\hline $\begin{array}{l}\text { Microgravity } \\
\text { Opportunity }\end{array}$ & Unlimited & Approx. $22 \mathrm{~s}$ & Approx. 9s & $\begin{array}{l}\text { Limited to mission } \\
\text { lifetime }\end{array}$ & $\begin{array}{l}\text { Limited to time on- } \\
\text { board the space station }\end{array}$ \\
\hline Cost & $\begin{array}{l}\text { Approx. } £ 6320 \text { (one-off } \\
\text { cost) }\end{array}$ & $\begin{array}{c}\text { Approx. } € 2700 \text { for } 12 \\
\text { parabolas }\end{array}$ & Affordable & $\begin{array}{l}\text { Approx. } £ 75000 \text { for a } \\
3 U \text { cubesat launch }^{\text {cost }}{ }^{d}\end{array}$ & $\begin{array}{l}\text { Approx. } \mathrm{f} 12 \text { million per } \\
\text { trip }{ }^{\text {e }}\end{array}$ \\
\hline Reconfigurability & Yes & Yes & Yes & No & $\begin{array}{l}\text { Yes but depending on } \\
\text { the available resources }\end{array}$ \\
\hline $\begin{array}{c}\text { Maximum } \\
\text { Manoeuvrability }\end{array}$ & $\pm 45^{\circ}$ & $\pm 360^{\circ}$ & $\begin{array}{l} \pm 360^{\circ} \text { (limited by } \\
\text { capsule size) }\end{array}$ & $\pm 360^{\circ}$ & $\pm 360^{\circ}$ \\
\hline Convenience to reset & No limitation & $\begin{array}{l}\text { Limited to number of } \\
\text { parabolic flight }\end{array}$ & $\begin{array}{l}\text { Limited to frequency } \\
\text { of "drop" session }\end{array}$ & $\begin{array}{l}\text { Within mission } \\
\text { lifetime }\end{array}$ & Within mission lifetime \\
\hline Minimal Hardware Class & COTS & COTS & COTS & Space-grade & $\begin{array}{c}\text { COTS with space station } \\
\text { constraints }\end{array}$ \\
\hline Experimental Area & $\begin{array}{c}\text { Normal lab area - no fix } \\
\text { constraint }\end{array}$ & $\begin{array}{l}100 \mathrm{~m}^{2} \text { with } \max \\
\text { height of } 2.5 \mathrm{~m}\end{array}$ & $\begin{array}{l}0.8 \mathrm{~m} \text { capsule } \\
\text { diameter with max } \\
\text { height of } 2.4 \mathrm{~m}\end{array}$ & $\begin{array}{c}\text { Spare }- \text { no area } \\
\text { constraint. Testbed } \\
\text { however constraint } \\
\text { by fairing size }\end{array}$ & $\begin{array}{l}\text { Approx. } 3-4 \mathrm{~m} \\
\text { diameter with the } \\
\text { longest module to be } \\
\text { approx. } 11 \mathrm{~m}^{\mathrm{f}}\end{array}$ \\
\hline $\begin{array}{c}\text { Gravity Disturbance } \\
\text { (Balancing } \\
\text { Requirement) } \\
\end{array}$ & Yes & No & No & No & - sprop - \\
\hline Number of Advantages & 5 & 4 & 3 & 3 & 2 \\
\hline $\begin{array}{c}\text { Number of } \\
\text { Disadvantages }\end{array}$ & 2 & 2 & 3 & 3 & 1 \\
\hline
\end{tabular}

"Data obtained from Specialty Components

${ }^{b}$ Data obtained from Air Zero $G$ of Novaspace and Avico

'Data obtained from ZARM Drop Tower of University of Bremen

¿Data obtained from SatMagazine article (http://www. satmagazine.com/story.php?number=602922274)

eApproximation on space station based on the average cost of a space tourist on-board ISS

'Area on space station acquired from Kibo module of ISS 


\section{B. Design Concept}

A related review on the design is made and from this, several design concepts are concluded and listed as below.

1) Earth-based space testing system.

2) Usage of an air bearing platform to emulate microgravity.

3) A testbed with minimal variation in the moment of inertia for $\mathrm{x}$ and $\mathrm{y}$ axis (axisymmetric shape).

4) Preventing the usage of inertial actuators such as CMG or reaction wheel and utilising the external actuators such as thrusters and magnetorquers to avoid centre of mass change during any actuation.

5) Preventing the usage of any spinning component as part of the hardware on top of the spherical air bearing to avoid gyroscopic disturbance.

6) A relatively compact and lightweight testbed to minimise the effect of gravity disturbance.

7) Utilising well-known RCP and HIL development techniques that is Matlab Simulink-xPC Target and/or LabVIEW FPGA for the algorithm development and testing tool.

As it was initially designed for the prolate spinner slew control algorithms another factor is considered. The $\lambda$ value is needed to be between range 0.15 and 0.20 . This $\lambda$ is referring to the ratio of the moment of inertia between the spin and the transverse axis. Ensuring this is crucial in order to have a suitable range for every developed slew algorithm to be tested.

\section{Modular Spinning TestBed Design}

The design of the testbed is developed based on the guidelines that have been determined in Sec. II and further trade-off analyses on each subsystem is detailed in the following sub-sections. A cubesat like approach has been adopted and the CAD model of the design can be referred to the following Fig. 1. From the model, the mass properties of the testbed are determined and listed as the following:

- Total weight $=42.39 \mathrm{~kg}$;

- $\mathrm{I}_{\mathrm{xx}}=9.4134 \mathrm{~kg} \cdot \mathrm{m}^{2}$;

- $\mathrm{I}_{\mathrm{yy}}=9.4150 \mathrm{~kg} \cdot \mathrm{m}^{2}$;

- $\mathrm{I}_{\mathrm{zz}}=1.8428 \mathrm{~kg} \cdot \mathrm{m}^{2}$;

- $\lambda=0.196$ (ratio between $\mathrm{I}_{\mathrm{zz}}$ and $\mathrm{I}_{\mathrm{xx}} / \mathrm{I}_{\mathrm{yy}}$ )

\section{A. On-Board Computer (OBC)}

The system design approach for the testbed adopts the RCPHIL system design architecture as mentioned in the design concept. The selection of a target $\mathrm{OBC}$ for this design approach depends on the type of RCP-HIL software that is used. As for the software, Matlab ${ }^{\circledR}$ Simulink-xPC Target is utilised. Any PC-compatible hardware with supported I/O can be used as the target hardware for this prototyping method. In term of physical factor, the following chart in Fig. 2 is used to help in the selection.

Ideally, a PC/104 form factor would be the best selection due to its size. However as the existing PCISA form factor is readily available, it is selected as the target hardware for the

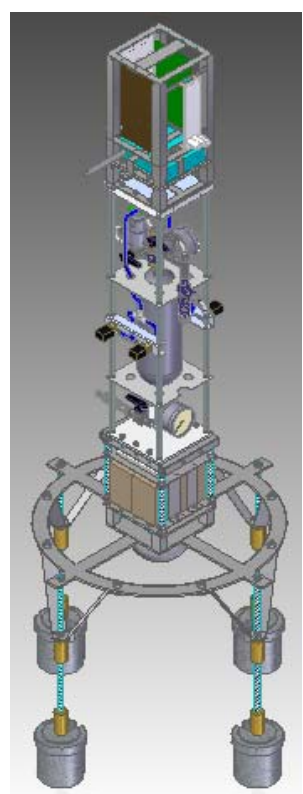

Fig. 1. CAD for the spinning testbed in prolate mode.

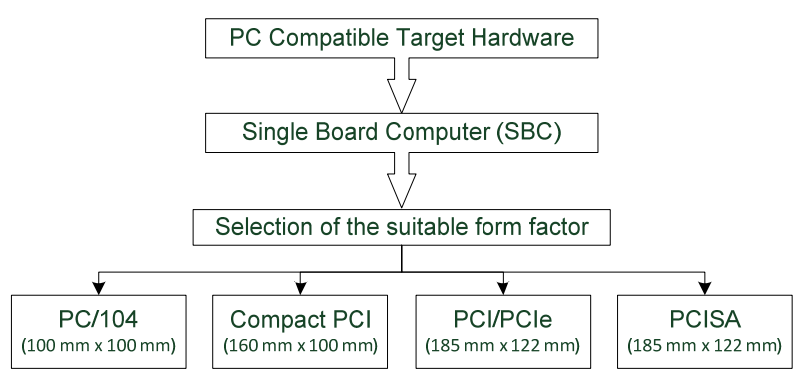

Fig. 2. Target $\mathrm{OBC}$ form factor selection.

testbed. Cuboid frame made from aluminum with a size of 15 $\mathrm{cm} \times 15 \mathrm{~cm} \times 20 \mathrm{~cm}$ is used to hold the hardware.

\section{B. Electrical Power Subsystem (EPS)}

Although the testbed is ground based testing system, the power supply from mains cannot be used as the hardware needed to be 'levitated' on the air bearing platform. Therefore, a power supplied by a battery is needed. The next factor to be determined is the voltage level for the operating voltage needed by the testbed. The crucial determinant will be the operating voltage needed by the $\mathrm{OBC}$ of the testbed.

The OBC selected need a 12 VDC nominal power supply hence, a battery with a nominal voltage of 12 VDC is used. A wide type of batteries can provide this kind of nominal voltage. For a portable design, Nickel-Cadmium, Nickel-Metal Hydride and Lithium-Ion would be chosen and among these three, Lithium-Ion is preferable due to its higher energy density [12]. Other than that, Lithium-Ion battery has the lowest cost among the three mentioned type with other advantages such as no memory effect, long shelf-life and rapid charge capability [13].

By having this 12 VDC nominal voltage, a regulated 3.3 VDC, $5 \mathrm{VDC}$ and $12 \mathrm{VDC}$ is achieved using the readily 
available DC-DC converter. The other subsystems are designed to operate within this available voltage supplies. Additional DC-DC converters are fitted to the EPS to cater for any additional need. Having the voltage busses determined, the next crucial factor is to calculate the needed battery capacity. The following Table 2 justifies the needed battery capacity.

TABLE 2

BATTERY CAPACITY CALCULATION

\begin{tabular}{|c|c|c|c|c|}
\hline Component & $\begin{array}{l}\text { Voltage } \\
\text { (V) }\end{array}$ & $\begin{array}{c}\text { Typical } \\
\text { Current (A) }\end{array}$ & $\begin{array}{l}\text { Operation } \\
\text { in } 1 \text { hour }\end{array}$ & Wh \\
\hline JUKI SBC & 12 & 1.7 & $100 \%$ & 20.4 \\
\hline PCI I/O Modules & 5 & 1.424 & $5 \%^{\mathrm{g}}$ & 0.356 \\
\hline $\begin{array}{l}\text { D-Link Wireless } \\
\text { Access Point }\end{array}$ & 5 & 2 & $100 \%$ & 10 \\
\hline Actuators & 12 & $0.4^{\mathrm{g}}$ & $5 \%^{\mathrm{g}}$ & 0.24 \\
\hline $\begin{array}{l}\text { Infrared (IR) } \\
\text { Markers }\end{array}$ & 1.6 & $0.3^{\mathrm{h}}$ & $100 \%$ & 0.48 \\
\hline Others & & & & $5^{g}$ \\
\hline \multicolumn{3}{|c|}{ Total } & & 36.47 \\
\hline \multicolumn{3}{|c|}{ Depth of Discharge (DoD) } & \multicolumn{2}{|c|}{$40 \%$} \\
\hline \multicolumn{3}{|c|}{ Actual Watt-hour (Wh) } & \multicolumn{2}{|c|}{$\begin{array}{c}36.47 / 0.4=91.19 \\
\text { Wh }\end{array}$} \\
\hline \multicolumn{3}{|l|}{ Capacity $^{i}$} & \multicolumn{2}{|c|}{$\begin{array}{c}91.19 \mathrm{Wh} / 12 \mathrm{~V} \approx \\
7.6 \mathrm{Ah}\end{array}$} \\
\hline
\end{tabular}

Notes:

${ }^{\mathrm{g}}$ Approximation;

${ }^{\mathrm{h}}$ Based on 5 Ir markers; and

Not taking the effect of ambient temperature

The following concludes the guideline of selecting the battery for the EPS of the testbed:

- $12 \mathrm{~V}$ nominal output voltage.

- 7-8 Ah of battery capacity.

- Can be contained within the $15 \mathrm{~cm} \times 15 \mathrm{~cm}$ area

Although the ideal solution is to use a light weight lithium based battery, a readily available lead acid battery that fits the criteria is used to keep the cost at the minimum.

\section{Communication Subsystem (COM)}

The selection of the COM for the testbed follows the similar concept as the OBC previously. As the system design approach has been fixed to an RCP-HIL strategy the COM is designed to cater for a link between the host $\mathrm{PC}$ and the target hardware for Matlab ${ }^{\circledR}$ Simulink-xPC Target. By default a wired communication via either a serial or TCP/IP protocol will be used for these two RCP-HIL techniques. Due to the requirement of 'levitating' the target on top of the air bearing platform, a wireless communication method is employed.

The OBC of the testbed is connected to a wireless access point (WAP) to form a wireless bridge between the target and the host PC of the Matlab ${ }^{\circledR}$ Simulink-xPC. A typical $2.4 \mathrm{GHz}$ wireless connection is used for this communication. An IP address is fixed for the target and is used as the ID for the host PC to connect with target in the RCP-HIL setting. The WAP is fitted in a frame with the base dimension of $15 \mathrm{~cm} \times 15 \mathrm{~cm}$. It is stacked together with the other subsystem within the 'levitated' testbed.

\section{Attitude Control Subsystem (ACS)}

As mentioned in the design concept, inertial actuator is not used for this purpose. As a result, a normal cold gas propulsion system is fitted. Normal compressed air is used as the propellant and it is stored in a miniature supply canister. This canister is connected to a miniature solenoid valve that acts as the thruster valve. Other than that the system is consisting of:

- A system for filling in or draining out the compressed air to and from the miniature canister.

- A pressure regulator to regulate the system pressure within the intended value.

- Pressure gauges to monitor the pressure.

- A relief valve for safety precaution.

The following Fig. 3 shows the block diagram for the propulsion subsystem.

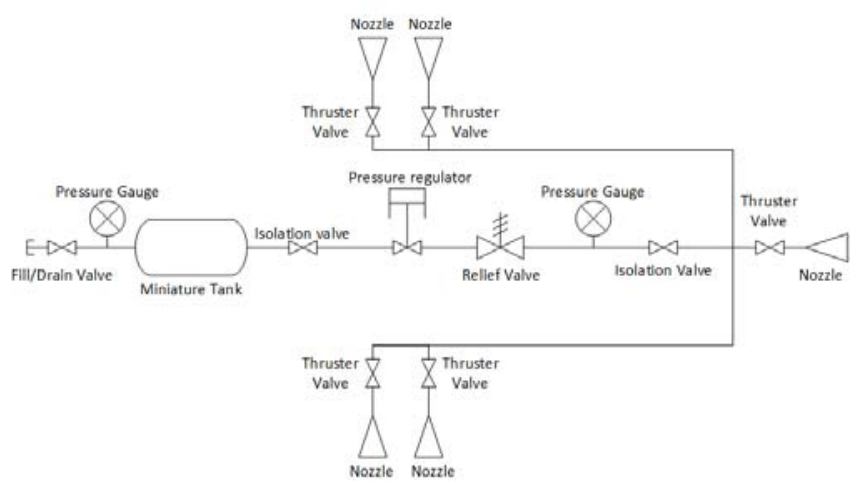

Fig. 3. Block diagram for the propulsion subsystem.

For the selection of the thruster valve, the size of it is needed to be small enough to be fitted into the intended frame work. A survey of a miniature solenoid valves that have been used as a thruster valve is done. This specific survey is revolving around the valves that have been developed for cubesat purposes. Comparison between these valves has been done in [14]. Seven cold gas thrusters have been reviewed in this comparison. However as these valves are aimed for a fight model, the price is high and it is unsuitable for ground testing purposes. One example is the Moog manufactured valve that has a price tag of about $£ 10 \mathrm{~K}$. Therefore, a general purpose miniature solenoid valve is considered.

The next trade-off done is for the size of the supply canister. As the volume of the compressed air needed to make the slew is dependent to the $\lambda$ value for the testbed itself, the selection process is started by selecting a canister with a size that can be fitted to the intended area. The flow rate is then calculated by using the known maximum operating operation differential (MOPD) and the flow coefficient equation as stated at the following (1).

$$
C_{v}=\frac{Q}{13.61 \times P_{\text {inlet }} \sqrt{\frac{1}{S G \times T}}}
$$




$$
\begin{aligned}
& C_{v}=\text { Flow coefficient } \\
& Q=\text { Flow rate in standard cubic feet per minute } \\
& P_{\text {inlet }}=\text { Pressure at the inlet of the valve psia } \\
& S G=\text { Specific gravity } \\
& T=\text { Temperature in degree Rankine }
\end{aligned}
$$

Equation 2 is then used to compare the needed volume of air with the volume of air stored in the tank. This equation is used due to the different pressure set between the stored air and the regulated pressure used for the thruster valve.

$$
V_{\text {storage }}=\frac{P_{\text {regulated }} \times V_{\text {needed }}}{P_{\text {storage }}}
$$

$$
\begin{aligned}
& V_{\text {storage }}=\text { Equivalent volume of air in the storage tank } \\
& P_{\text {storage }}=\text { Air pressure in the storage tank } \\
& V_{\text {needed }}=\text { Volume of air needed at the regulated pressure } \\
& P_{\text {regulated }}=\text { Pressure regulated before the thruster valve }
\end{aligned}
$$

For the testbed, a supply canister with a volume within 500 $\mathrm{cm}^{3}-1000 \mathrm{~cm}^{3}$ is selected.

\section{E. Attitude Determination Subsystem (ADS)}

The testbed uses a low cost monocular vision system for the attitude determination as stated in the introduction section. This vision system utilizes infra-red (Ir) LED marker positioned around the test platform. The only needed work to be done before the vision system can be used is to determine the location and perform the needed calibration. The system implementation can be referred further in [5]. In this section the position of the needed IrLED markers is shown and the calibration process is described.

These IrLEDs is positioned around the testbed according to the rules mentioned in [5]. The following Fig. 4 shows the location of the four co-planar markers and the virtual triangles made by these markers to suit the identification algorithm run by the vision system. The fifth marker is place on top of the prolate frame at about 1 meter from the center of bearing.

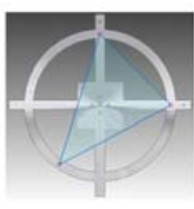

Largest area

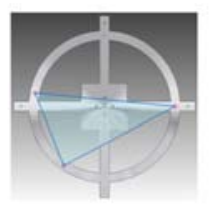

$2^{\text {nd }}$ largest

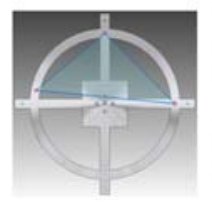

$3^{\text {rd }}$ largest

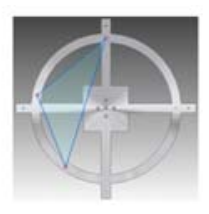

Smallest
Fig. 4. Location of the 4 co-planar markers and virtual triangles area made.

For the calibration, the guideline is shown in the following steps.

1) Camera configuration matrix, $R_{c a m}$ in the vision system is initialised to identity matrix.

2) The attitude of the testbed is determined in term of the yaw (rotation along $y$-axis) and the pitch (rotation along $\mathrm{x}$-axis) angle using the available 2-axis inclinometer.

3) The roll (rotation along the z-axis) angle of the testbed is acquired by rotating the testbed to the reference point determined as roll equal to 0 degree.

4) The attitude acquired by the vision system is recorded at the same time as the reading of the inclinometer is taken with roll rotated to a reference roll of 0 degree.

5) Both set of attitude acquired using the inclinometer and the vision system are then used as the input of the readily developed Matlab® Simulink model.

6) $R_{\text {cam }}$ is then calculated by the model. The calculated $R_{\text {cam }}$ will be used to change the initial $R_{\text {cam }}$ of the vision system that has been set to identity matrix initially.

7) Vision system is ready to be used with the new testbed provided the location of both the testbed and the camera has not been changed.

After the calibration is done following the guideline mentioned, the camera configuration matrix, $\mathrm{R}_{\mathrm{cam}}$ for the testbed is as below.

$$
R_{\text {cam }}=\left[\begin{array}{ccc}
0.9978 & -0.0505 & -0.0425 \\
0.0469 & 0.9956 & -0.0815 \\
0.0464 & 0.0794 & 0.9958
\end{array}\right]
$$

\section{Testbed Performance \& Verification}

Before any work can be done using the testbed, a balancing process is crucial to make sure that the disturbances resulted by the gravity is reduced to its minimum. The first step of balancing done to the testbed is balancing the platform in its $\mathrm{x}$ and $y$ axis direction. This procedure is done by making sure that the centre of mass (CoM) for the testbed in the $\mathrm{z}$-axis is below the centre of bearing $(\mathrm{CoB})$ so that the prolate structure (as it is for the moment) do not tumble. The attitude of the platform is checked and verified using the monocular vision system in term of its balanced mode in $\mathrm{x}$ and $\mathrm{y}$ axis.

Having done that, the $\mathrm{z}$-axis balancing procedure is done next. The counterweights below are moved upwards every 1 $\mathrm{mm}$ until the threshold of where the prolate structure is about to tilt and tumble, is determined. The length of the expose threaded pole below the first small brass counterweight underneath the ' $\mathrm{S}$ ' shape support for the testbed base is used to indicate the needed position of the counterweights to balance it along the z-axis. For the current prolate configuration the length of this expose threaded pole is $\mathbf{1 2 5} \mathbf{~ m m}$. Fig. 5 shows the current length of the mentioned expose threaded pole.

Once the testbed is balanced, the CAD model is updated with the additional small weights and the current length of the counterweights below the base to produce the updated mass properties. Fig. 6 shows the latest CAD model with the estimated moment of inertia that is used throughout the remainder of this section. Fig. 7 shows the detail locations of the fine balancing weights on top of the testbed base. 


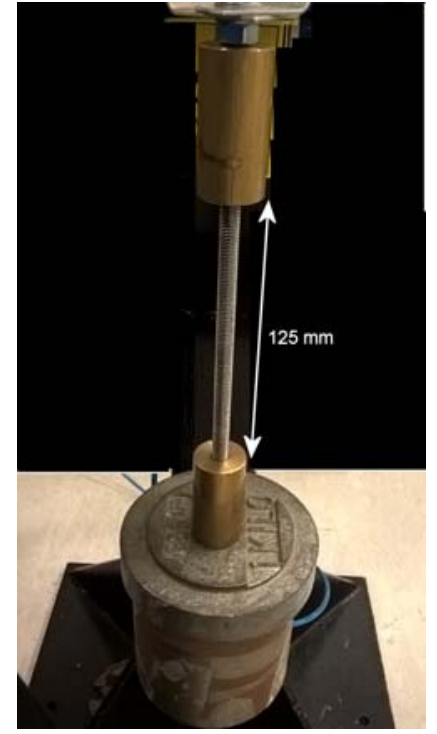

Fig. 5. The length to indicate the position of the counterweight for balancing purposes.

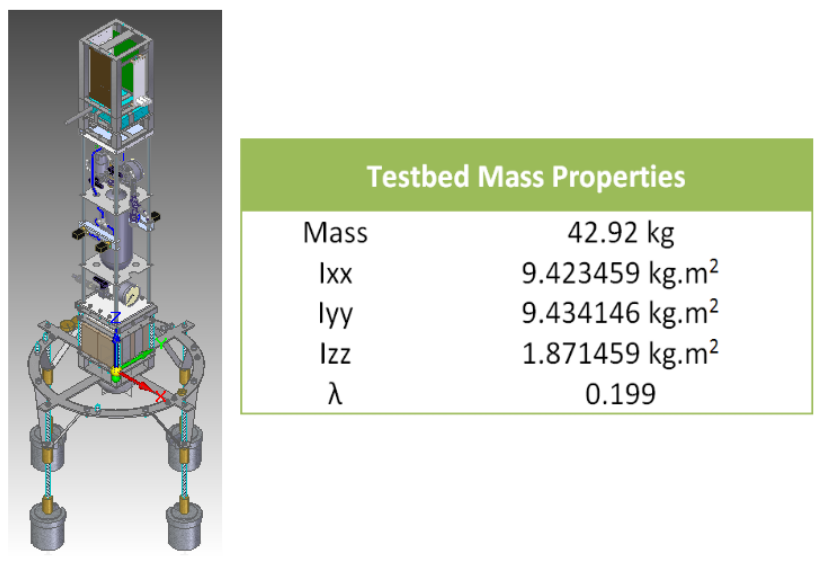

Fig. 6. Updated testbed $\mathrm{CAD}$ with corresponding moment of inertia.

The confirmation of thrust produced by the developed cold gas propulsion system is determined as the first step for the testbed verification after it is balanced. This is crucial as the actual thrust produced is needed to be known before any algorithm can be tested. Theoretically, with the set operating pressure of $50 \mathrm{psi}$ and the valve orifice size of $2 \mathrm{~mm}$ as mentioned in the specification, a thrust of approximately 1.08 $\mathrm{N}$ can be produced.

However, this calculated value can dramatically change if the actual orifice size differs from the mentioned value in the specification. For example if the actual orifice size is reduced from $2 \mathrm{~mm}$ to $1 \mathrm{~mm}$, the thrust that can be produced will only be approximately $0.27 \mathrm{~N}$.

In order to measure the thrust produced by the testbed, a simple spinning up procedure is done. The spinning up actuators is turned on for a period of $10 \mathrm{~s}$ and this spins up the testbed. The angular velocity of the testbed is then measured using the vision system. By having the updated moment of inertia information of the testbed together with the plot of the
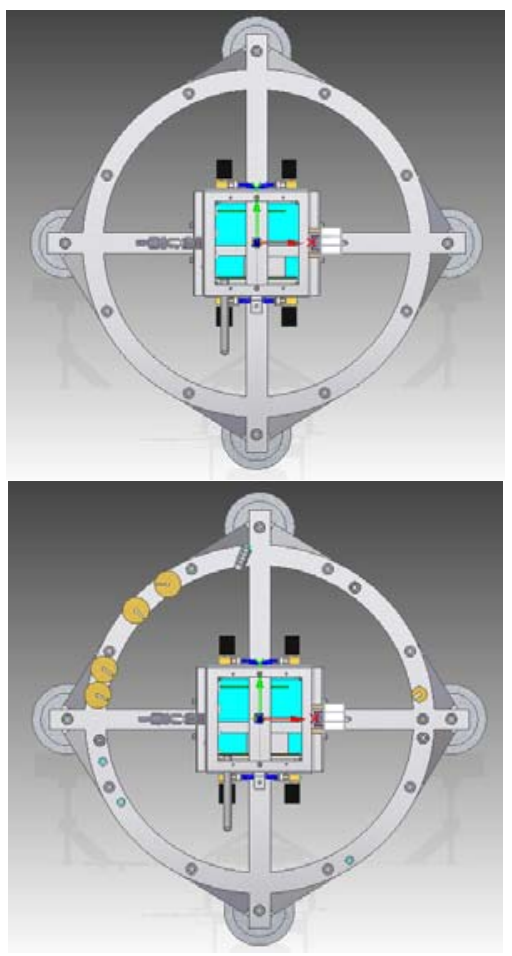

Fig. 7. Platform without fine weights (above) and with it (below).

measured angular velocity for a set period of time, the thrust produced is calculated. The following Fig. 8 shows the angular velocity plot acquired.

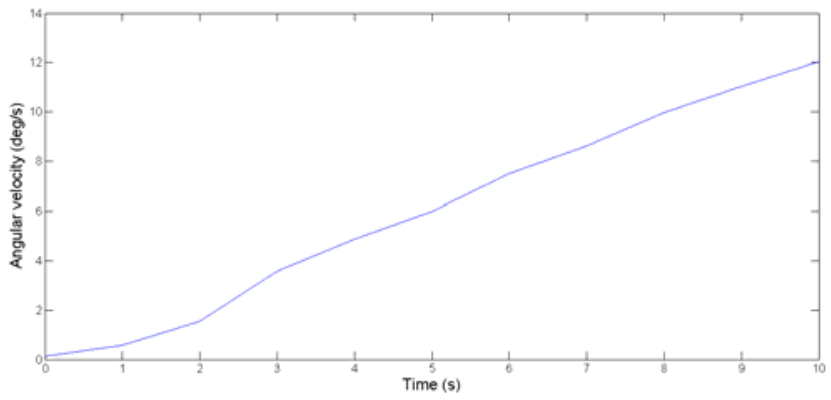

Fig. 8. Testbed spin up test velocity plot.

From the plot, the angular acceleration of the testbed is deduced. Comparing this with the calculated torque figure, the produced thrust is calculated. Using the mentioned method, a thrust of $\mathbf{0 . 2 5} \mathbf{N}$ is foreseen for each thruster. This value is vastly smaller than the theoretically value of $1.08 \mathrm{~N}$. As discussed, inaccurate information on the actual size of the orifice is believed to be the reason of this big difference.

Once the produced thrust is verified, a free spin test is done to the testbed. In an ideal condition, the spin axis position in reference inertial (RI) frame should remain the same over the period of time. However a deviation with a maximum value of 3.14 degree is observed during this free spin test. The plot can be referred to the following Fig. 9. In this plot, the location of the spin axis is represented by the dots and this free spin manoeuvre is observed for a period of 30 minutes.

This outcome is expected when a hardware testbed is used. An ideal condition is impossible to be achieved as there are 


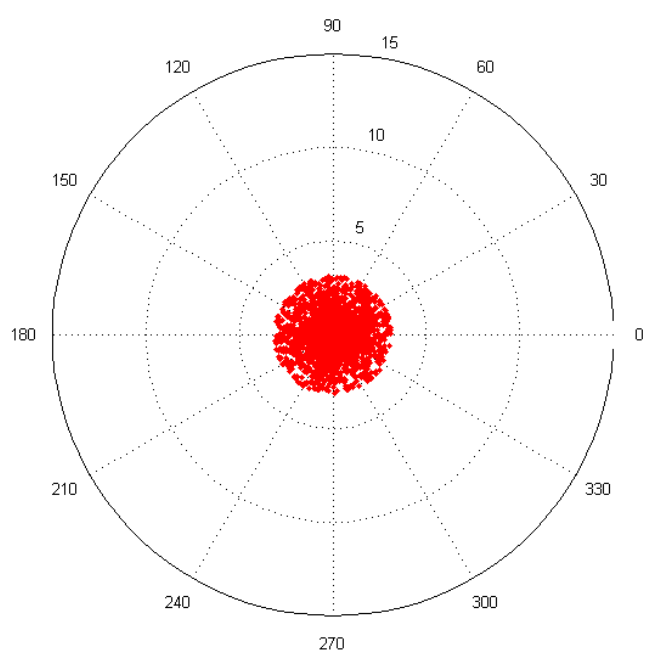

Fig. 9. Top view of the spin axis of the testbed during free spin.

many factors that go against this such as the asymmetric shape of the testbed, gravity disturbance torque due to balancing and non-rigidity of the testbed physical configuration. This result however shows a vast improvement from the testbed predecessor that can only run the free spin test for a time period of 200s with the maximum angle of deviation of 15 degree.

After running the free spin test, the profile of the existed disturbance torque is produced. The first disturbance torque to be profiled is the frictional torque of the air bearing. Although in theory, this torque is quantified as zero but in the real implementation, it is not the case. This is proven by the angular velocity plot of the testbed free spin that can be seen in the following Fig. 10.

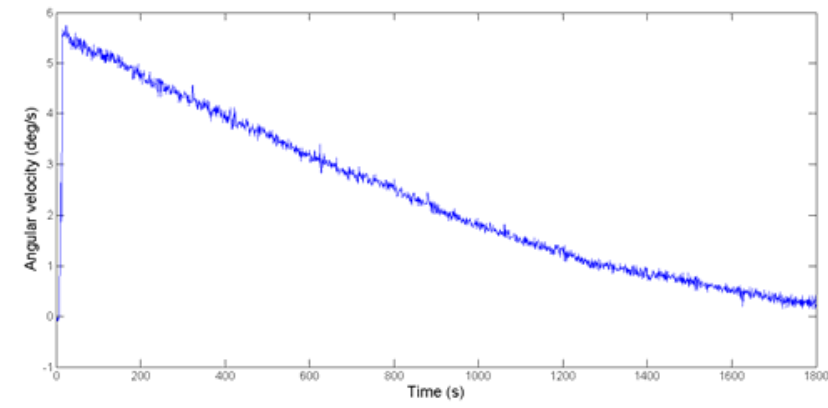

Fig. 10. Angular velocity plot for 30 minutes testbed free spin.

Spin up thrusters are turned on for 5 seconds and the testbed angular velocity in z-axis is observed. In an ideal condition, the angular velocity should not decrease as the testbed sits on top of a frictionless air bearing. However it is not the case where the angular velocity is reduced from $5.6 \mathrm{deg} / \mathrm{s}$ to almost zero in approximately 30 minutes. The frictional torque calculated based on the plot is $\mathbf{1 . 0 2 9} \times \mathbf{1 0}^{-4}$ N.m.

Other than the frictional torque, the gravity disturbance torque is also profiled for the testbed. During the balancing process, the testbed was found to be tumbled over when the counterweights were move up $1 \mathrm{~mm}$ from the current $125 \mathrm{~mm}$ counterweights' location. This has been used to assume that the offset between $\mathrm{CoB}$ and $\mathrm{CoM}$ in the z-axis is below $1 \mathrm{~mm}$. The following Matlab ${ }^{\circledR}$ Simulink model shown in Fig. 11 is then used to calculate the maximum gravity disturbance torque that the testbed is experiencing together with its estimate of the period for the pendulum shape movement.

Gravity disturbance torque of the testbed depends on the angle deviation from its equilibrium position. The following (3) calculates the gravity disturbance torque oscillation frequency, $\omega_{0}$ with the angle deviation, $\theta$ value set to have close to no influence to the testbed's pendulum motion.

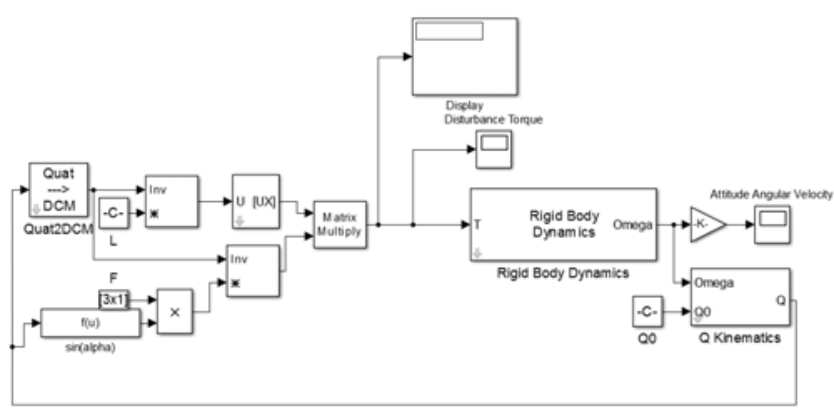

Fig. 11. Simulink model used to calculate the gravity disturbance torque.

$$
\omega_{0}=\sqrt{\frac{g / d_{z}}{I_{t} / m_{\mathrm{z}}^{2}}} \quad \begin{aligned}
& g=\text { gravity acceleration } \\
& d_{z}=\text { CoM offset along z-axis } \\
& I_{t}=\text { transverse MoI } \\
& m=\text { testbed mass }
\end{aligned}
$$

Equation 3 is derived with an assumption that the deviation angle is small enough that lead to the following condition shown by (4).

$$
\sin \theta \cong \theta
$$

As a result, the only significant factor that contributes to the gravity disturbance torque would only be dependent on the testbed physical properties. However, using the Simulink model developed in Fig. 11, the magnitude of this gravity disturbance torque is profiled through a series of deviation angle from 0 to 10 degrees. The following Fig. 12 shows the profile while Fig. 13 shows the torque oscillation frequency through the same series of deviation angle from the testbed equilibrium position.

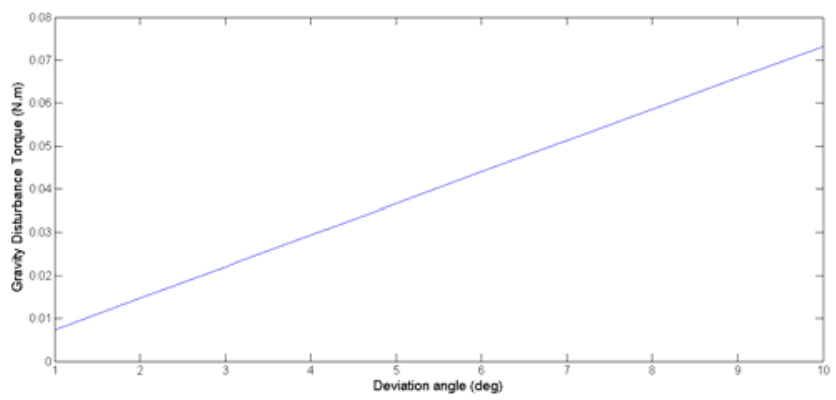

Fig. 12. Plot for gravity disturbance torque through a series of deviation angle. 


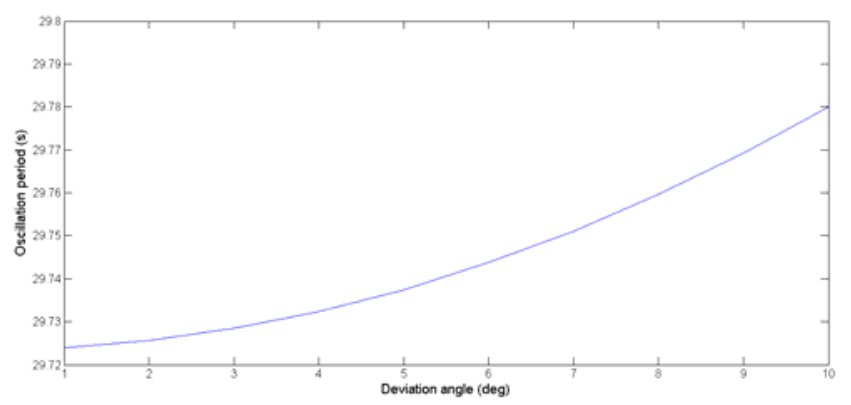

Fig. 13. Plot for pendulum oscillation period through a series of deviation angle.

As the free spin test indicates that the maximum deviation angle for the testbed from its equilibrium position is $\mathbf{3 . 1 4}$ degree, the maximum gravity disturbance torque that the testbed encountered is $\mathbf{0 . 0 2 3 0 8}$ N.m with an estimated period of oscillation of $\mathbf{2 9 . 7 2 9} \mathrm{s}$. Fig. 14 shows the plot of the testbed attitude angular velocity during the pendulum motion for the angle deviation of 3.14 degree from which the period mentioned before is estimated.

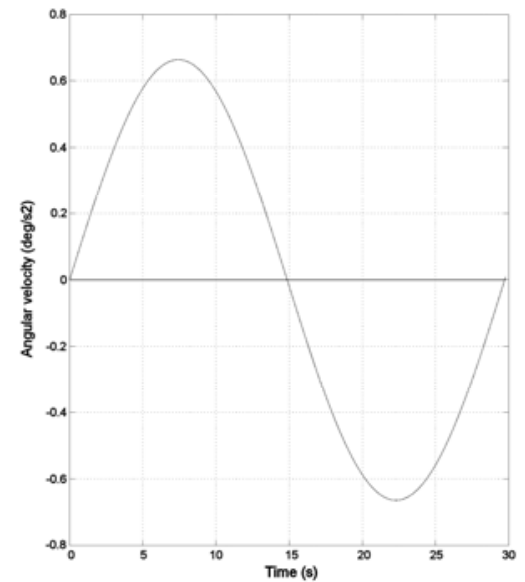

Fig. 14. Estimated period for the pendulum motion of the testbed.

\section{REFERENCES}

[1] Raus, R., Gao, Y., Wu, Y., and Watt, M., "Analysis of state-of-the-art single-thruster attitude control techniques for spinning spacecraft," Acta Astronautica, Vol. 76, Jul-Aug 2012, pp. 60-78, doi:10.1016/j.actaastro.2012.02.014.

[2] Wu, Y., Gao, Y., Raus, R., and Watt, M., "Single-thruster attitude control algorithms for spin-stabilised spacecraft: Tradeoff study," Journal of Guidance, Control, and Dynamics, Vol. 35, No.4, Jul. 2012 pp. 1143-1157, doi:10.2514/1.55619.

[3] Raus, R., Gao, Y., Wu, Y., and Watt, M., "Robustness analyses of attitude slew manoeuvres for spinning penetrator spacecraft," $63^{\text {rd }}$ International Astronautical Congress, IAC-12-C1.8.4, Naples, Italy, 2012.

[4] van der Ha, J.C., "Models for Rhumb-Line Attitude Maneuvers and Error Propagation Effects," Journal of Guidance, Control, and Dynamics, Vol.29, No. 6, 2006, pp. 1384-1394.

[5] Wu, Y., Gao, Y., Lin, J., Raus, R., Zhang, S., and Watt, M., "Low-Cost High-Performance Monocular Vision System for Air Bearing Table Attitude Determination," Journal of Spacecraft and Rockets, Vol. 51, Jan. 2014, pp. 66-75 doi:10.2514/1.A32465.

[6] Schwartz, J.L., Peck, M.A., and Hall, C.D., "Historical Review of AirBearing Spacecraft Simulators," Journal of Guidance, Control, and Dynamics, Vol. 26, No. 4, 2003, pp. 513-522.
The disturbance torque profiled for the testbed is crucial as it is needed to be included in the verification model before any test can be worked out. Other than this two mentioned torques, another factor such as the torque caused by the air drag, can contribute to this profile but looking at how the testbed is arrange at the moment, this torque is foreseen as insignificant.

The final verification done to the testbed is the integration of the testbed with a different payload. The aim of this step is to prove the modularity of the testbed physically and systematically. A gripper for the Chinese Space Station mission [15] is integrated on top of the testbed arrangement. This robotic gripper is then interfaced to the testbed OBC via serial connection. Several commands are sent to the gripper using Simulink ${ }^{\circledR}$ Real-Time and the output is observed.

Although this is just a simple integration procedure, the objective of having an easy to integrate testbed is demonstrated through this step.

\section{CONCLUSION \& RECOMMENDATION}

Modular testbed for spin-stabilised spacecraft has been developed and profiled through a series of test as mentioned in the previous section. Through the development, constraints of operating such a testbed were also identified. The implementation of the testbed also proves that COTS product can be used to build this platform hence producing a low cost solution. However, this implementation can still be improved to create a better solution apart from the addition of several features as mentioned in Sec. II. Nevertheless, it is proven that the interest in a spin-stabilised spacecraft is still valid inspite the popularity of its counterpart, the three-axis stabilisation technique.

[7] Raschke, C., Roemer, S., and Grossekatthoefer, K., "Test bed for verification of attitude control system," Astro- und Feinwerktechnik Adlershof $\mathrm{GmbH}, 2011$.

[8] Kenyon, S., et al., "STRaND-1: Use of a \$500 Smartphone as the Central Avionics of a Nanosatellite," 62 ${ }^{\text {nd }}$ International Astronautical Congress, IAC-11.B4.6B.8, Cape Town, SA, 2011.

[9] Ure, N.K., Y.B. Kaya, and G. Inalhan. The Development of a Software and Hardware-in-The-Loop Test System for ITU-PSAT II Nano Satellite ADCS, IEEE Aerospace Conference. 2011.

[10] Al-Majed, M.I. and B.N. Alsuwaidan. A New Testing Platform for Attitude Determination and Control Subsystems: Design and Applications, IEEE/ASME International Conference on Advanced Intelligent Mechatronics. 2009. Suntec Convention and Exhibition Center, Singapore.

[11] Yavuzoglu, E., et al. Verification of Control Moment Gyroscopes Based Attitude Control Systems for Agile Satellite Missions, 5th International Conference on Recent Advances in Space Technologies (RAST). 2011. Istanbul, Turkey.

[12] Li, W., et al. The Research on Lithium-ion Battery's Rapid Charging Strategy with Ant-Cycle System. in International Conference on Mechatronics and Automation. 2012. Chengdu, China.

[13] Linden, D. and T.B. Reddy, Handbook of Batteries 3rd Edition. 2002: McGraw-Hill.

[14] Mueller, J., et al., Survey of Propulsion Option for Cubesats. JANNAF, 2010(57th).

[15] Robosat for Chinese Tiangong Mission. 